\title{
Study of charge control and gate tunneling in a ferroelectric-oxide-silicon field effect transistor: Comparison with a conventional metal-oxide- silicon structure
}

\author{
Yih-Yin Lin a) \\ Department of Electrical Engineering and Computer Science, University of Michigan, \\ Ann Arbor, Michigan 48109-2122 \\ Yifei Zhang \\ Applied Physics Program, University of Michigan, Ann Arbor, Michigan 48109-2122 \\ Jasprit Singh \\ Department of Electrical Engineering and Computer Science, University of Michigan, \\ Ann Arbor, Michigan 48109-2122 \\ Robert York and Umesh Mishra \\ Department of Electrical and Computer Engineering, University of California, \\ Santa Barbara, California 93106-9560
}

(Received 14 July 2000; accepted for publication 18 October 2000)

\begin{abstract}
It is known that conventional metal-oxide-silicon (MOS) devices will have gate tunneling related problems at very thin oxide thicknesses. Various high-dielectric-constant materials are being examined to suppress the gate currents. In this article we present theoretical results of a charge control and gate tunneling model for a ferroelectric-oxide-silicon field effect transistor and compare them to results for a conventional MOS device. The potential of high polarization charge to induce inversion without doping and high dielectric constant to suppress tunneling current is explored. The model is based on a self-consistent solution of the quantum problem and includes the ferroelectric hysteresis response self-consistently. We show that the polarization charge associated with ferroelectrics can allow greater controllability of the inversion layer charge density. Also the high dielectric constant of ferroelectrics results in greatly suppressed gate current. (C) 2001 American Institute of Physics. [DOI: 10.1063/1.1332425]
\end{abstract}

\section{INTRODUCTION}

Modern microelectronic success depends critically on field effect transistors based upon the metal-oxide-silicon (MOS) structure. However, as device dimensions scale down, several important problems are expected to arise. Large oxide capacitance, and consequently thin gate oxide, will be required to invert the surface to sufficient sheet charge and to reduce subthreshold current, avoiding shortchannel effects. However, it is known that at oxide thickness of $30 \AA$ or below, direct tunneling becomes the dominant transport mechanism. In addition, doping fluctuation ${ }^{1}$ is expected to create serious problems as channel lengths shrink to approximately $500 \AA$. For example, only ten dopant atoms are contained in a $0.1 \mu \mathrm{m}$ channel for the implant dosage of $10^{12} \mathrm{~cm}^{-2}$ at a depth of $0.01 \mu \mathrm{m}$, possibly resulting in random distribution.

Recently several important developments have occurred which suggest that the problems identified above can be addressed by new structures based on polar materialsemiconductor combinations. These developments are:

(1) In the $\mathrm{AlGaN} / \mathrm{GaN}$ system it has been shown that heterostructure field effect transistors can be fabricated with very large sheet charge density without the use of dopants. ${ }^{2,3}$

${ }^{a)}$ Electronic mail: yylin@engin.umich.edu
This has been made possible because of the very large polarization charge at the $\mathrm{AlGaN} / \mathrm{GaN}$ interface arising from spontaneous polarization and strain induced piezoelectric effect. This polarization charge can reach values as high as 5 $\times 10^{13} \mathrm{~cm}^{-2}$ and with a proper design has been shown to produce free carrier sheet charge of a similar value - all without any dopants.

(2) It is also well known that ferroelectrics like barium strontium titanate (BST) can have very high polarization charge $\left(\sim 10^{14} \mathrm{~cm}^{-2}\right) .{ }^{4}$ This charge may be developed during growth or can be induced by an external electric field. Additionally (unlike $\mathrm{AlGaN}$ ), BST and other ferroelectrics have a very large dielectric constant (relative dielectric constant can be in the hundreds) ${ }^{5}$ Moreover, ferroelectrics have been now grown on silicon ${ }^{6-8}$ and are becoming important for dynamic random access memory applications. ${ }^{9-12} \mathrm{Re}-$ cently high quality field effect transistors with $\mathrm{SrTiO}_{3}$ gate dielectrics have been reported with greatly suppressed gate current. ${ }^{13}$ Also a ferroelectric has been deposited on $\mathrm{SiO}_{2} / \mathrm{Si}$ and the capacitance-voltage $(C-V)$ measurements show a high quality structure. ${ }^{8}$

Two questions arise when we examine these developments: (1) What are the charge control characteristics of a ferroelectric- $\mathrm{SiO}_{2} / \mathrm{Si}$ structure where the inversion charge is induced by the polarization charge of the ferroelectric material? (2) How influential are large dielectric constants of the 
ferroelectrics (for our study we use BST) in suppressing gate tunneling current? In this article we address these questions.

\section{THEORETICAL FORMALISM}

In order to study the charge control in a $\mathrm{BST}-\mathrm{SiO}_{2} / \mathrm{Si}$ MOS structure and to examine gate tunneling, we need to examine the band profile in detail by carrying out a selfconsistent quantum treatment. We have extended a previously published model ${ }^{2,3}$ to do so. Results shown here are based on a self-consistent charge control model which is capable of introducing multiple epilayers with material properties that can vary continuously. This model first obtains the potential profile in a MOS structure by solving the Schrödinger equation and Poisson equation self-consistently. Solution of the Schrödinger equation yields the confined charge terms in the Poisson equation which, in turn, determine the potential profile. The potential profile is then fed back into the Poisson equation until the solution goes to convergence. Since we want to develop a generic model that can be applied to ferroelectric and nonferroelectric oxides, we include the polarization of the material in our model. The ferroelectric polarization is included by the introduction of a spontaneous charge as the boundary condition at the ferroelectrics/ oxide interface

$$
\epsilon_{1} \mathbf{E}_{1}+\mathbf{P}_{1}=\epsilon_{2} \mathbf{E}_{2}
$$

where $\epsilon_{1}$ and $\epsilon_{2}$ are the dielectric constants and $\mathbf{E}_{1}$ and $\mathbf{E}_{2}$ are the electric fields at the interface of ferroelectrics and oxide. $\mathbf{P}_{1}$ is the polarization field due to switching dipoles, and is a function of the field history in ferroelectric materials. The magnitude of saturated polarization hysteresis loop, $P_{\text {sat }}$, is defined by

$$
P_{\text {sat }}^{+}(\mathbf{E})=P_{s} \tanh \left[\left(\mathbf{E}-\mathbf{E}_{\mathbf{c}}\right) / 2 \delta\right],
$$

where

$$
\delta=\mathbf{E}_{\mathbf{c}}\left[\ln \left(\frac{1+P_{r} / P_{s}}{1-P_{r} / P_{s}}\right)\right]^{-1} .
$$

The positive superscript in Eq. (2) refers to the forward biasing of the loop. $\mathbf{E}$ is the applied field and $\mathbf{E}_{\mathbf{c}}$ is the coercive field of the ferroelectric field. $P_{r}$ and $P_{s}$ are the remanent and spontaneous polarization, respectively. The magnitude of saturated polarization in the reverse loop, $P_{\text {sat }}^{-}$, is defined as

$$
P_{\text {sat }}^{-}(\mathbf{E})=-P_{\text {sat }}^{+}(-\mathbf{E}) \text {. }
$$

In our simulation, we assume that the quantity $P_{s}$ can be related to a polarization charge $\sigma$ by an electron charge, and we assume a value of $10^{14}$ charges $/ \mathrm{cm}^{2}$ for $\sigma, 200 \mathrm{kV} / \mathrm{cm}$ for the coercive field and 0.8 for the ratio of $P_{r}$ to $P_{s}$. We find that $C-V$ measurements on structures can allow one to extract these parameters by fitting the experimental results.

The one-dimensional (1D) Poisson equation can be written as

$$
\frac{d^{2}}{d z^{2}} E_{c}(z)=-\frac{\rho(z)}{\epsilon(z)}
$$

where $E_{c}$ is the conduction band profile, $\rho$ is the total charge density, and $\epsilon$ is the dielectric constant in different regions of the device. The total charge density $\rho$ includes the doping charge, free charge of the hole and electron, and quantumconfined charge

$\rho(z)=q\left[N_{d}^{*}(z)-N_{a}^{*}(z)-n_{\text {free }}+p_{\text {free }}-\sum_{i} n_{i} \psi_{i}^{*}(z) \psi_{i}(z)\right]$,

where $N_{d}^{*}$ and $N_{a}^{*}$ are the effective doping concentrations, $n_{\text {free }}$ and $p_{\text {free }}$ are the free carrier concentrations, and the sum is over $i$ two-dimensionally (2D) confined subbands of which normalized envelope functions are $\psi$ and in which the occupation is $n_{i}$. The free carrier density can be obtained from

$$
n_{\text {free }}(z)=N_{c} F_{1 / 2}\left[\frac{E_{f}-E_{c}(z)}{k_{B} T}\right],
$$

where $N_{c}$ is the effective density of states and $F_{1 / 2}$ is a halforder Fermi integral. The 2D gas in the quantum well region of Eq. (6) can be determined by solving the Schrödinger equation for the subband envelope functions and their occupations. The $z$-dependent part of the Schrödinger equation is strictly separable from the in-plane part of the equation which gives us extended Bloch-like states. The 1D Schrödinger equation can be written using the perpendicular part of the effective mass tensor as follows:

$$
\frac{d^{2}}{d z^{2}} \psi_{n}(z)+\frac{2 m_{w}}{\hbar^{2}}\left[E_{n}-v(z)\right] \psi_{n}(z)=0,
$$

where $m_{w}$ is the electron effective mass along the quantum confinement direction.

When the envelope function and subband energy levels are solved from the Schrödinger equation, the subband occupation can be calculated from the first-order Fermi integral. The occupation for electrons can be described as

$$
n_{i}=\frac{m_{i} k_{B} T}{\pi \hbar^{2}} \ln \left[1+\exp \left(\frac{E_{i}-E_{f}}{k_{B} T}\right)\right]
$$

where $m_{i}$ is the in-plane effective mass of the $i$ th subbands.

Once the band profile is calculated the gate to channel electron tunneling probability $T$ is obtained from the equation $^{14}$

$$
T=\exp \left[-\frac{1}{\hbar} \int_{x_{1}}^{x_{2}}\left[8 m^{*}(V(x)-E)\right]^{1 / 2} d x\right],
$$

where $E$ is the energy of the particle, $m^{*}$ is the electron effective mass of the barrier, $V(x)$ is the potential barrier along $x$ direction, and $x_{1}$ and $x_{2}$ are points where $E$ $=V(x)$.

\section{RESULTS AND DISCUSSION}

The structure simulated by us is shown in the upper part of Fig. 1(a). It consists of a layer of BST (or another ferroelectric) on a standard $\mathrm{SiO}_{2} / \mathrm{Si}$ structure. The intermediate $\mathrm{SiO}_{2}$ layer is included since it is likely that growth of the ferroelectric may be easier on a thin $\mathrm{SiO}_{2}$ layer. ${ }^{8}$ Also this way the inversion layer is at the high quality $\mathrm{Si} / \mathrm{SiO}_{2}$ inter- 
(a)

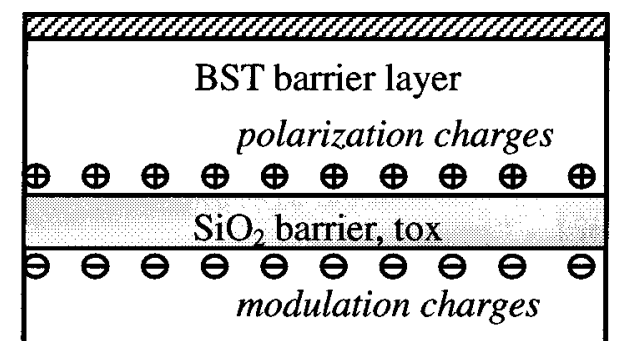

p-type silicon substrate

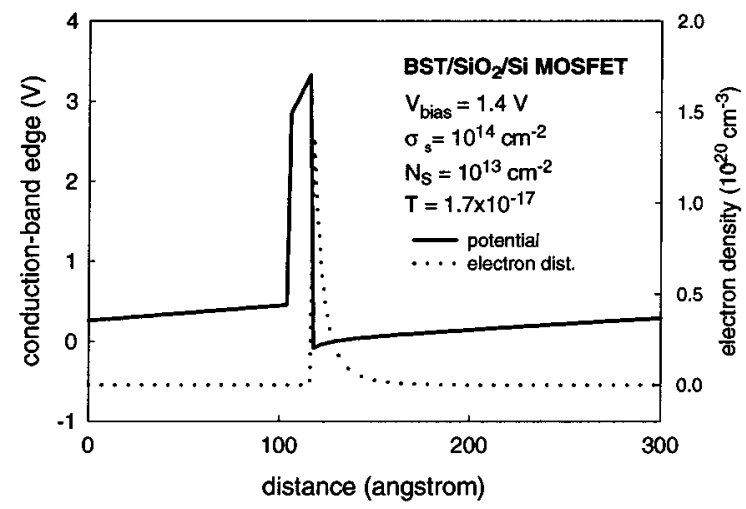

(b)

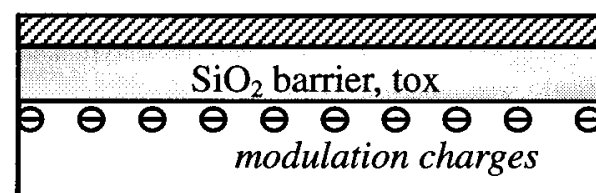

p-type silicon substrate

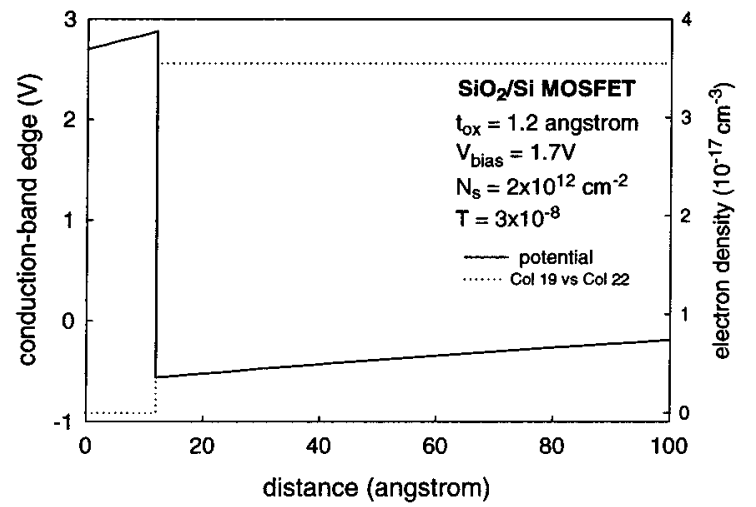

FIG. 1. (a) The $\mathrm{BSTi} / \mathrm{SiO}_{2} / \mathrm{Si}$ structure for simulation; results for the band profile, charge density and tunneling probability $T$ for an inversion charge density of $10^{13} \mathrm{~cm}^{-2}$. The polarization charge $(\sigma)$ is assumed to be $10^{14}$ $\mathrm{cm}^{-2}$. (b) A conventional MOS structure and the band profile and electron charge distribution for the device biased at the same value as in (a). The induced charge density is small compared with ferroelectric-oxide based structure, but tunneling probability is high.

face. As far as gate tunneling suppression is concerned, the thin $\mathrm{SiO}_{2}$ layer has negligible effects. The $p$-type silicon shown in Fig. 1(a) is doped at $1 \times 10^{17} \mathrm{~cm}^{-3}$. In the upper part of Fig. 1(b) we show a conventional MOS structure. The
TABLE I. Material parameters of the simulated $\mathrm{BSTi} / \mathrm{SiO}_{2}$ structure.

\begin{tabular}{lccclc}
\hline \hline & Symbol & Unit & BST value & $\mathrm{SiO}_{2}$ & Si 2D value \\
\hline Energy gap & $E_{g}$ & $\mathrm{eV}$ & 3.4 & 8 & 1.12 \\
Dielectric constant & $\epsilon$ & $\epsilon_{0}$ & 300 & 3.9 & 11.9 \\
Electron effective mass & $m_{e}$ & $m_{0}$ & 0.19 & 0.50 & 1.08 \\
Hole effective mass & $m_{h}$ & $m_{0}$ & 0.45 & 0.50 & 0.49 \\
\hline \hline
\end{tabular}

following assumptions are made for our modeling studies: (i) BST can be grown with spontaneous polarization; (ii) the band gap of BST is approximately $3.4 \mathrm{eV} ;{ }^{15}$ (iii) the carrier masses in BST are shown in Table I; however, this assumption has little impact on the results; (iv) perfect interfaces are assumed in this study, but the model itself can allow us to set up the interface states and examine their effects. It should be noted that $\mathrm{BST}$ composition must have high $\mathrm{Ba} / \mathrm{Sr}$ ratio to exhibit spontaneous polarization. ${ }^{16}$

The thickness of BST film is allowed to vary to examine its effects on the tunneling probability. The material parameters are given in Table I. The conduction band discontinuity is assumed to be $50 \%$ of the band discontinuity at the $\mathrm{BST} / \mathrm{SiO}_{2}$ and $\mathrm{SiO}_{2} / \mathrm{Si}$ interfaces, and the Schottky barrier is taken to be $1.7 \mathrm{eV} .^{17}$

A typical band diagram for $n$-channel BST-oxide metaloxide-silicon field effect transistors (MOSFET) is shown in Fig. 1(a). The device is biased to obtain an inversion charge of $10^{13} \mathrm{~cm}^{-2}$. As can be seen, it is possible to obtain a very high sheet charge without doping or large external field because of the large polarization charge. In contrast, Fig. 1(b) shows the band diagram of a conventional MOS with equivalent oxide thickness as the device in Fig. 1(a). Although biased at the same voltage, the induced charge density is a factor of 5 smaller.

In Fig. 2(b) we show the capacitance-voltage $(C-V)$ relations for the BST based structure. We see that the gate has a good control on the inversion charge. The device behaves as if we have a MOS with an effective oxide thickness which is

$$
d_{(\mathrm{eff})}=\frac{\epsilon_{\mathrm{ox}}}{\epsilon_{\mathrm{BST}}} d_{\mathrm{BST}}+d_{\mathrm{ox}} .
$$

We have also shown in Fig. 2(b) counterclockwise $C$ - $V$ curves due to the hysteresis polarization. This hysteresis nature of ferroelectric materials can be described by Eqs. (2), (3), and (4), and shown schematically in Fig. 2(a) as a function of external field. The hysteresis of $C-V$ characteristic has been demonstrated experimentally with a $\mathrm{SBT} / \mathrm{CeO}_{2} / \mathrm{SiO}_{2} / \mathrm{Si}$ MOSFET device. ${ }^{8}$ The $C-V$ curve of a conventional MOSFET of the equivalent oxide thickness is shown in Fig. 2(c). The increase of capacitance in the inversion region is sluggish and the threshold voltage is larger compared with the ferroelectric-based MOSFET due to low inversion charge density. The comparison of Figs. 2(b) and 2(c) suggests that the ferroelectric-based MOSFET has a larger transconductance, which can improve the device's performance. 

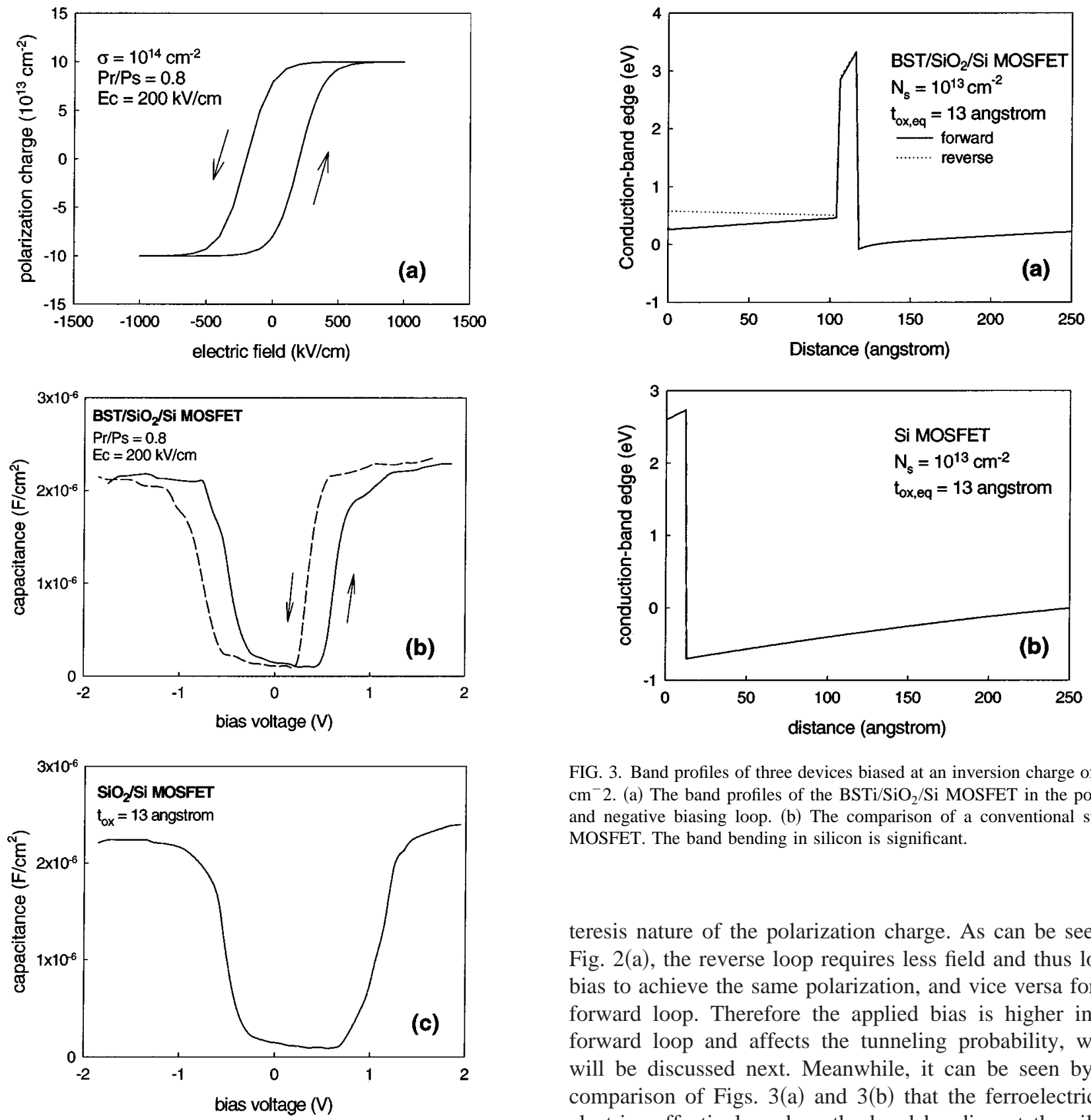

FIG. 2. (a) The ferroelectric polarization as a function of the history of the electric field. The simulated device has a saturation polarization charge of $10^{14} \mathrm{~cm}^{-2}$, a coercive field of $200 \mathrm{kV} / \mathrm{cm}$, and the ratio of remanent to spontaneous polarization is 0.8 . (b) The $C$ - $V$ relation for the ferroelectricoxide MOSFET. The effect of the hysteresis curve for the polarization charge causes the $C$ - $V$ curve to shift as shown. (c) The $C$ - $V$ curve of the conventional MOSFET with equivalent oxide thickness.

Figure 3 illustrates the band profile of three cases biased at an inversion charge of $10^{13} \mathrm{~cm}^{-2}$ : (i) forward voltage applied on a $\mathrm{BSTi} / \mathrm{SiO}_{2} / \mathrm{Si} \mathrm{MOSFET}$ in a hysteresis loop, (ii) reverse voltage applied on the same device in case (i), and (iii) a conventional MOSFET of equivalent oxide thickness. Cases (i) and (ii) are shown in Fig. 3(a) while case (iii) is shown in Fig. 3(b). It can be seen from Fig. 3(a) that the band profiles in silicon are identical in cases (i) and (ii) for the same amount of charges; however, the conduction-band profile in the BST region differs significantly due to the hys-

FIG. 3. Band profiles of three devices biased at an inversion charge of $10^{14}$ $\mathrm{cm}^{-}$2. (a) The band profiles of the $\mathrm{BSTi} / \mathrm{SiO}_{2} / \mathrm{Si}$ MOSFET in the positive and negative biasing loop. (b) The comparison of a conventional silicon MOSFET. The band bending in silicon is significant.

teresis nature of the polarization charge. As can be seen in Fig. 2(a), the reverse loop requires less field and thus lower bias to achieve the same polarization, and vice versa for the forward loop. Therefore the applied bias is higher in the forward loop and affects the tunneling probability, which will be discussed next. Meanwhile, it can be seen by the comparison of Figs. 3(a) and 3(b) that the ferroelectric dielectrics effectively reduce the band bending at the silicon interface.

In Fig. 4 we show how the tunneling probability from the gate to the channel changes as a function of oxide thickness in the conventional MOS device and the BST-based structure. The tunneling probability is calculated in each case for an inversion layer carrier density of $10^{13} \mathrm{~cm}^{-2}$. In the conventional oxide-based transistor, the tunneling probability is not very sensitive to thickness in this range of thicknesses considered. The probability is in the range of $10^{-6}$ which is very high. In the BST based structure, due to the larger thickness of the BST region (even though the effective oxide thickness is the same) the tunneling probability is greatly suppressed. As we can see from the calculation, the tunneling is negligible once the effective thickness reaches about $14 \AA$. Reduced tunneling probability in MOSFET by high-dielectric gate materials like $\mathrm{SrTiO}_{3}$ has also been shown empirically. ${ }^{13}$ Referring to Fig. 3(a), it can be understood that the tunneling probability is lower in the reverse 


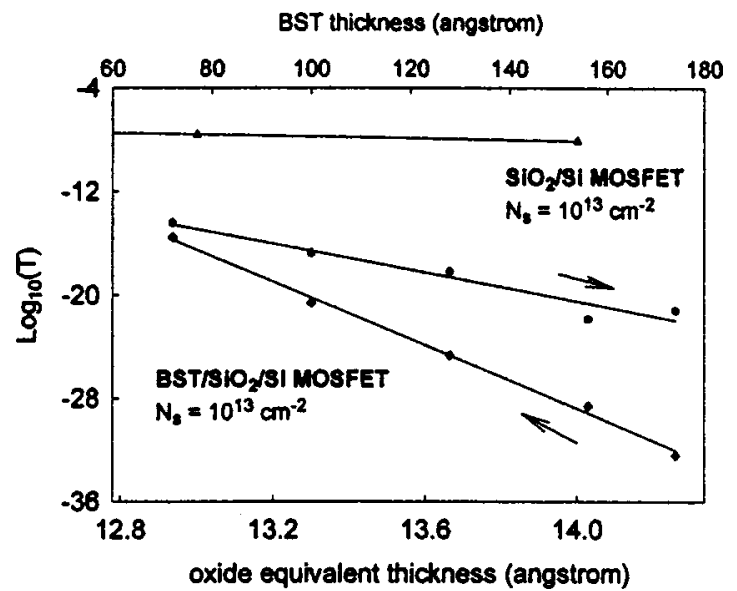

FIG. 4. Comparison of the tunneling probability of BST-oxide and oxidebased MOS structures as a function of film thickness.

biasing loop. With the same inversion charge, the potential barrier is higher in the reverse loop and thereby suppresses tunneling.

Ferroelectric materials also exhibit other characteristics dependent on thickness, such as remanent polarization and even coercive field in very thin films. ${ }^{18}$ The magnitude of remanent polarization is proportional to the thickness of ferroelectric thin film, therefore inducing more inversion charges as film thickness increases. The threshold voltage is consequently reduced, capable of building depletion-mode MOSFETs without additional doping by adjusting film thickness.

It is important to note that the study shown here is done to show the potential of ferroelectric-semiconductor based devices. The technology which will allow such devices to be made is still in its infancy. Issues of interface charge, accurate values for the polarization charge and its control, dielectric constant values etc. need to be resolved before such devices can be exploited. However, our study shows the enormous benefits that can result from developing the ferroelectric-semiconductor technology.

\section{SUMMARY}

We have reported results on a one-dimensional charge control model and estimation of gate tunneling probability for ferroelectric-oxide MOSFETs. We have examined the modulation charge, tunneling probability and the band profile as a function of spontaneous charges, and applied voltage. We see that in comparison to the conventional MOS this structure has almost five times more induced-charge density and the tunneling probability is greatly suppressed. The model developed by us is quite generic and can be applied to ferroelectric and nonferroelectric system.

\section{ACKNOWLEDGMENTS}

This work has been supported by Grant Nos. F001681 and F000629 from the U.S. Office of Naval Research. The work at UC Santa Barbara has been supported by the ONR IMPACT program and the DARPA FAME program. Discussions with Professor J. Speck of UC Santa Barbara have been extremely valuable.

${ }^{1}$ Y. Taur and T. Ning, Fundamentals of Modern VLSI Devices (Cambridge University Press, Cambridge, 1998), p. 200.

${ }^{2}$ Y. Zhang and J. Singh, J. Appl. Phys. 85, 587 (1999).

${ }^{3}$ Y. Zhang, I. P. Smorchkova, C. R. Elsass, S. Keller, J. P. Ibbetson, S. Denbaars, U. K. Mishra, and J. Singh, J. Appl. Phys. 87, 7981 (2000).

${ }^{4}$ T. Chen, V. Balu, S. Katakam, J. Lee, and J. C. Lee, IEEE Trans. Electron Devices 46, 2304 (1999).

${ }^{5}$ P. Padmini, T. R. Taylor, M. J. Lefevre, A. S. Nagra, J. S. Speck, and R. A. York, Appl. Phys. Lett. 75, 3186 (1999).

${ }^{6}$ K. Aizawa and H. Ishiwara, Jpn. J. Appl. Phys., Part 1 33, 5178 (1994).

${ }^{7}$ J. Yu, Z. Hong, W. Zhou, G. Cao, J. Xie, and X. Li, Appl. Phys. Lett. 70, 490 (1997).

${ }^{8}$ Y. T. Kim and D. S. Shin, Appl. Phys. Lett. 71, 3507 (1997).

${ }^{9}$ Y. Ishioka et al., in Proceedings of the International Electron Devices Meeting (IEEE, New York, 1995), p. 903.

${ }^{10}$ S. Yamamichi et al., in Ref. 9, p. 119.

${ }^{11}$ K. P. Lee et al., in Ref. 9, p. 907.

${ }^{12}$ E. Fujii et al., in Ref. 9, p. 267.

${ }^{13}$ K. Eisenbeiser, J. M. Finder, Z. Yu, J. Ramdani, J. A. Curless, J. A. Hallmark, R. Droopad, W. J. Ooms, L. Salem, S. Bradshaw, and C. D. Overgaard, Appl. Phys. Lett. 76, 1324 (2000).

${ }^{14}$ S. Gasiorowicz, Quantum Physics (Wiley, New York, 1974), p. 86.

${ }^{15}$ J. Robertson and W. L. Warren, Mater. Res. Soc. Syno, Oric. 361, 123 (1995).

${ }^{16}$ K. Abe and S. Komatsu, J. Appl. Phys. 77, 6461 (1995).

${ }^{17}$ C. S. Hwang, B. T. Lee, C. S. Kang, K. H. Lee, H. Cho, H. Hideki, W. D. Kim, S. I. Lee, and M. Y. Lee, J. Appl. Phys. 85, 287 (1999).

${ }^{18}$ K. Amanuma, T. Mori, T. Hase, T. Sakuma, A. Ochi, and Y. Miyasaka, Jpn. J. Appl. Phys., Part 1 32, 4153 (1993). 